\title{
FORMAÇÃO DO FISIOTERAPEUTA PARA O CUIDADO NO CONTEXTO DA ATENÇÃO PSICOSSOCIAL
}

\author{
Jean Carlos Müller da Silva Bizarro ${ }^{1}$ \\ Eliara Ten Caten Martins ${ }^{2}$
}

\section{RESUMO}

Este estudo tem como objetivo verificar a formação e o preparo do fisioterapeuta para o cuidado na atenção psicossocial. Trata-se de pesquisa exploratória, descritiva, de abordagem qualitativa e transversal, com aplicação de questionário estruturado aos fisioterapeutas da atenção psicossocial no município de São José/SC e análise dos currículos da graduação de Fisioterapia. Os dados coletados foram analisados utilizando-se a análise de conteúdo e desvelaram a existência de três fisioterapeutas atuando no cuidado em saúde mental no município. Todos foram inseridos na atenção psicossocial mediante concurso público inespecífico, a partir do qual jamais imaginavam atuar com saúde mental. Evidenciou-se que os fisioterapeutas não são preparados ao longo da graduação para esse cuidado em saúde mental. A inserção do fisioterapeuta na atenção psicossocial, apesar de ser imprescindível, ainda não é uma realidade estabelecida e necessita progredir por meio da reformulação dos currículos da graduação em Fisioterapia.

Palavras-chave: Atenção psicossocial. Fisioterapia. Educação superior.

\section{Como citar este documento - ABNT}

BIZARRO, Jean Carlos Müller da Silva; MARTINS, Eliara Ten Caten. Formação do fisioterapeuta para o cuidado no contexto da atenção psicossocial. Revista Docência do Ensino Superior, Belo Horizonte, v. 10, e023959, p. 1-14, 2020. DOI: https://doi.org/10.35699/2237-5864.2020.23959.

\footnotetext{
${ }^{1}$ Universidade Federal de Santa Catarina (UFSC), Florianópolis, SC, Brasil. ORCID ID: https://orcid.org/0000-0002-8971-8320. E-mail: drjeanbizarro@hotmail.com ${ }^{2}$ Instituto de Ensino Superior da Grande Florianópolis (IESGF), São José, SC, Brasil. ORCID ID: https://orcid.org/0000-0001-7498-125X. E-mail: eliara.martins@hotmail.com
} 


\section{FORMACIÓN DE FISIOTERAPEUTAS PARA EL CUIDADO EN EL CONTEXTO DE LA ATENCIÓN PSICOSOCIAL}

\section{RESUMEN}

Este estudio tiene como objetivo verificar la formación y preparación del fisioterapeuta para el cuidado en la atención psicosocial. Se trata de una investigación exploratoria, descriptiva, con enfoque cualitativo y transversal, con la aplicación de un cuestionario estructurado a fisioterapeutas en atención psicosocial en la ciudad de São José/SC y análisis de los planes de estudio de pregrado en Fisioterapia. Los datos recolectados fueron analizados mediante análisis de contenido y revelaron la existencia de tres fisioterapeutas que trabajan en salud mental en el municipio. Todos ellos fueron incluidos en la atención psicosocial a través de exámenes públicos no especificados, en los que nunca se imaginaron trabajar con salud mental. Se hizo evidente que los fisioterapeutas no son preparados durante la carrera para esta atención de salud mental. La inserción del fisioterapeuta en la atención psicosocial, a pesar de ser fundamental, aún no es una realidad establecida y necesita avanzar a través de la reformulación de los planes de estudio de pregrado en Fisioterapia.

Palabras clave: Atención psicosocial. Fisioterapia. Educación superior.

\section{PHYSIOTHERAPIST TRAINING FOR CARE IN THE CONTEXT OF PSYCHOSOCIAL ATTENTION}

\section{ABSTRACT}

This study aims to verify the training and preparation of the physiotherapist for care in psychosocial attention. This research is exploratory, descriptive and presents a qualitative and transversal approach, with the application of a structured questionnaire to physiotherapists in psychosocial care in the city of São José/SC, and analysis of the undergraduate Physiotherapy curricula. The collected data were analyzed using the content analysis, revealing the existence of three physiotherapists working in mental health care in the city. All of them were included in psychosocial care through a non-specific public tender, in which they never imagined working with mental health. It became evident that physiotherapists are not prepared during graduation for this mental health care. The insertion of the physiotherapist in psychosocial attention, despite being essential, is not yet an established reality and needs to progress through the reformulation of the undergraduate curricula in Physiotherapy.

Keywords: Psychosocial attention. Physiotherapy. Higher education. 


\section{INTRODUÇÃO}

O despontar do Sistema Único de Saúde (SUS), a partir da 8a Conferência Nacional de Saúde, em 1986, configura-se como um marco histórico de ampliação do acesso à saúde para a população brasileira. A mobilização social por melhores condições de saúde permitiu que, a partir de 1988, o SUS se consolidasse como política pública, com a promulgação da Lei 8.080/90, conhecida como Lei Orgânica da Saúde (BRASIL, 1988, 1990a, 1990b; COSTA, 2002; NÓBREGA, C. et al., 2010).

Consoante ao processo de construção do SUS, houve uma grande mobilização, por parte dos trabalhadores da saúde mental, para reestruturação do modelo manicomial vigente. A década de 1980 foi marcada por uma eclosão de manicômios e a mercantilização da loucura, com elevados índices de internações manicomiais, onde os indivíduos denominados como loucos eram cerceados do convívio social. A justificativa para tal ação era a garantia da ordem e prevenção da perturbação social proveniente da mendicância e inatividade dos sujeitos alienados. As mobilizações resultaram no surgimento do primeiro Centro de Atenção Psicossocial (CAPS), no Rio de Janeiro, em 1987 (PITTA, 2011; RIBEIRO SCHNEIDER et al., 2013).

Os CAPS surgem como modelos substitutivos aos manicômios e expandem-se pelo país com o propósito de integrar uma rede de atenção à saúde mental. Corroborando essa reestruturação do sistema, em 2001, foi promulgada a Lei 10.216 , que previu as diretrizes para a efetiva implantação da Reforma Psiquiátrica e a consequente extinção dos manicômios existentes (BRASIL, 2005).

A articulação de políticas públicas de saúde torna-se cada vez mais evidente e prestigiosa, uma vez que o modelo de redes favorece o cuidado articulado entre os diferentes pontos de atenção em saúde. Nesse contexto, em 2011, por meio da Portaria 3.088, foi implantada a Rede de Atenção Psicossocial (RAPS) com objetivo de fortalecer o foco preventivo, promotor e curativo na assistência à saúde mental, por meio das ações de uma equipe multiprofissional (BRASIL, 2013).

O recurso terapêutico mais empregado na atenção psicossocial é o farmacológico, com o uso de medicações psicotrópicas. Essas medicações acarretam inúmeros efeitos colaterais, entre os quais se destacam ataxia, disartria, redução da capacidade psicomotora, astenia muscular, alterações no ritmo cardíaco, tremores periféricos, dentre outros (BRAGA et al., 2016).

A Fisioterapia é uma ciência da Saúde que atua de forma integrada na equipe multidisciplinar, desde a prevenção até o tratamento de distúrbios cinético-funcionais em órgãos e sistemas do corpo humano, provocados por alterações genéticas, traumas ou doenças adquiridas. Justifica-se, assim, a necessidade do fisioterapeuta no cuidado aos 
indivíduos no âmbito da atenção psicossocial, uma vez que os distúrbios cinético-funcionais causados pelo tratamento farmacológico são frequentes (CASTRO; CIPRIANO; MARTINHO, 2006; DELIBERATO, 2002; MAGALHÃES; RIBEIRO, 2020).

A RAPS torna-se um marco na ampliação do acesso e reorientação do cuidado em saúde mental, de modo singular, contínuo e integral. Reitera-se, assim, a necessidade de inserção da fisioterapia na RAPS, uma vez que são conspícuas as alterações osteomioarticulares, em razão da evolução da doença e da ampla carga de medicações utilizadas pelos indivíduos em sofrimento psíquico. Todavia, o modelo medicalocêntrico ainda é uma realidade muito prevalente, abnegando outras possibilidades de recursos terapêuticos não farmacológicos (BRAGA et al., 2016; BÜCHELE et al., 2006).

Apesar de a inserção da fisioterapia no cuidado em saúde mental ser indispensável, ainda é incipiente, menos da metade dos municípios brasileiros contam com a inserção da fisioterapia na atenção primária (MAGALHÃES; RIBEIRO, 2020; NÓBREGA, M. et al., 2017; PAULI; CAMPOS, 2016; SOUZA; SANTOS, 2017; TAVARES; COSTA; DRIUSSO, 2018). E, possivelmente, devido a essa incipiência, os cursos de graduação em Fisioterapia ainda enfrentam dificuldades na oferta de formação em saúde mental.

A existência da fisioterapia, enquanto profissão no Brasil, é recente e, de acordo com Ribeiro e Flores-Soares (2015), os próprios gestores dos sistemas de saúde desconhecem a atuação da fisioterapia. Soma-se a isso a ausência de preparo para o cuidado em saúde mental durante a formação acadêmica dos fisioterapeutas resultando em insegurança por parte dos egressos para atuar na atenção psicossocial. É ao longo da graduação que o fisioterapeuta adquire competências e habilidades que o qualificam para a atuação profissional, de modo que o não desenvolvimento dessas competências afeta diretamente o preparo desse profissional para atuar no contexto da atenção psicossocial (MAGALHÃES; RIBEIRO, 2020; MARTINHAGO; OLIVEIRA, 2012; RIBEIRO; FLORES-SOARES, 2015; SILVA, E., 2015;).

A relevância deste estudo justifica-se pela escassez de estudos científicos que abordem a formação e o preparo dos fisioterapeutas para o cuidado em saúde mental, a partir de sua inserção na atenção psicossocial. Há necessidade de fomentar dados que evidenciem a importância da atuação da fisioterapia na atenção psicossocial e da adequada formação dos fisioterapeutas para o cuidado em saúde mental. Esta pesquisa tem como objetivo verificar a formação e o preparo do fisioterapeuta para atuação na atenção psicossocial num município do Sul do Brasil.

\section{MÉTODO}

Trata-se de estudo exploratório e descritivo com delineamento qualitativo e transversal. Para o seu desenvolvimento foi identificada a estrutura da RAPS no município de São José. Ele está localizado na região da Grande Florianópolis e é o quarto município mais antigo do 
estado de Santa Catarina, contando com uma população estimada de 209.804 habitantes, conforme dados do Instituto Brasileiro de Geografia e Estatística - IBGE (PMSJ, 2020).

Após a identificação da estrutura da RAPS no município, realizou-se contato telefônico com cada instituição para verificar a existência de fisioterapeuta no respectivo local. A partir da identificação da inserção de fisioterapeuta nos locais investigados, verificou-se quais desses profissionais realizavam atendimentos a pacientes em sofrimento psíquico.

Evidenciou-se a existência de dez pontos da RAPS no município, sendo três CAPS (um do tipo I - destinado ao atendimento de transtornos mentais em regiões com mais de 15 mil habitantes; um do tipo II - destinado ao atendimento de transtornos mentais em regiões com mais de 70 mil habitantes; e um do tipo $A D$ - destinado ao tratamento de transtornos pelo uso de álcool e outras drogas), um Centro de Referência em Saúde Mental - CRSM, quatro Núcleos de Apoio à Saúde da Família - NASF, um Instituto de Psiquiatria - IPq - e um Centro de Psiquiatria e Dependência Química - Instituto São José. Os CAPS, o CRSM e os NASF são de gerência municipal; o IPq é de gerência estadual e o Instituto São José é uma instituição de caráter privado.

Dos dez pontos levantados, constatou-se a existência de um fisioterapeuta em cada NASF, totalizando quatro, e três fisioterapeutas no IPq. Do público alvo inicial de sete fisioterapeutas, quatro foram excluídos por não atenderem aos critérios de inclusão, que eram realizar atendimentos em saúde mental nos limites do município de São José e fazer parte de um dos pontos da RAPS.

A coleta dos dados com os três participantes do estudo ocorreu por meio de entrevista com roteiro semiestruturado contemplando dados sociodemográficos (idade e sexo), forma de inserção na RAPS (concurso público, contrato temporário ou regime CLT), tempo de formado (em anos), local de formação (instituição pública ou privada), titulação (graduação, especialização, mestrado, doutorado ou pós-doutorado) e preparo para o cuidado em saúde mental (cursos de formação específica para a área ou experiências prévias).

A entrevista ocorreu de forma individual e em um local reservado no próprio ambiente de trabalho dos participantes, respeitando a disponibilidade de cada um para responder à entrevista, sendo que cada uma delas durou cerca de 15 minutos. A coleta ocorreu no mês de agosto de 2018 e o anonimato dos participantes foi garantido mediante a utilização de códigos alfanuméricos F1, F2 e F3 (F de Fisioterapeuta e 1, 2, 3 correspondentes à ordem de entrevista). As entrevistas foram transcritas em tempo real pelos pesquisadores, conforme os participantes respondiam às questões do roteiro semiestruturado.

Numa segunda etapa realizou-se a busca pelas grades curriculares dos cursos de graduação em Fisioterapia ofertados por instituições de ensino superior localizadas na Grande Florianópolis. A busca ocorreu a partir das grades curriculares disponíveis nos sites das 
instituições de ensino, e resultou em seis instituições de ensino superior com oferta do curso de Fisioterapia e com grade curricular disponível em seus sites. As grades curriculares disponíveis foram integralmente lidas sendo identificada a oferta de disciplina específica de saúde mental em uma instituição.

A análise dos dados coletados seguiu os pressupostos da análise temática de conteúdo em três etapas sequenciais. Assim, após pré-análise, exploração do material, tratamento e interpretação dos resultados emergiram duas categorias temáticas: forma de inserção dos fisioterapeutas na RAPS e formação dos fisioterapeutas para o cuidado em saúde mental.

O presente trabalho é um dos pilares do macroprojeto intitulado Atuação da Fisioterapia na Rede de Atenção Psicossocial num município do Sul do Brasil, aprovado pelo Comitê de Ética e Pesquisa da Universidade Paulista, sob parecer consubstanciado $n^{\circ} 2.716 .073$, respeitando os preceitos éticos estabelecidos pela Resolução 466/12 do Conselho Nacional de Saúde, cuja coleta de dados se iniciou somente após sua aprovação.

\section{RESULTADOS E DISCUSSÃO}

\section{Caracterização dos participantes}

O resultado da pesquisa revelou que o município de São José conta com dez pontos que compõem a estrutura da RAPS, sendo oito pontos pertencentes à estrutura municipal, um ponto da estrutura estadual e um ponto da rede privada. Dentre os oito pontos da RAPS sob gerência municipal, encontram-se um CRSM que funciona em caráter ambulatorial e não dispõe de fisioterapeuta; um CAPS tipo I, um CAPS tipo II e um CAPS tipo AD, todos sem inserção de fisioterapeuta em seu quadro de profissionais; e quatro NASF que funcionam como estruturas de apoio às equipes da Estratégia de Saúde da Família (ESF) nas Unidades Básicas de Saúde (UBS), sendo que cada NASF conta com um fisioterapeuta, entretanto, nenhum deles atua com atendimentos em saúde mental.

Em contrapartida, no ponto da RAPS sob competência da gestão estadual situa-se o IPq, que é classificado como um centro de referência no tratamento de transtornos mentais e também no tratamento da dependência química. A instituição tem caráter hospitalar, cujo tratamento consiste na internação dos sujeitos em sofrimento psíquico. Nesse ponto da RAPS encontram-se três fisioterapeutas que realizam atendimentos em saúde mental.

Nesse contexto, a caracterização dos sujeitos participantes da pesquisa está descrita na Tabela 1, em que se percebe predomínio de profissionais do sexo masculino, contradizendo o que geralmente se encontra na área da Saúde (AMORIM et al., 2017; BIZARRO, 2016; NÓBREGA, M. et al., 2017; SILVA, N. et al., 2013; SOUZA; SANTOS, 2017). 
Evidenciou-se que dois dos três participantes possuem um tempo de formado significativo, o que corrobora os dados encontrados na pesquisa de Bizarro (2016) ao traçar o perfil dos profissionais da RAPS de diferentes estados do país. Outro ponto que se assemelha a essa pesquisa é o fato de a maioria dos participantes possuir pós-graduação, porém nenhum deles em área correlata à saúde mental, o que demonstra a ausência de formação para o cuidado no contexto da atenção psicossocial.

\begin{tabular}{ccc}
\hline Variável & Categoria & $\mathbf{n ~ ( 3 )}$ \\
\hline Idade em anos & $25-35$ & 1 \\
Sexo & $35-45$ & 2 \\
\cline { 2 - 3 } Tempo de formado (em anos) & Feminino & 1 \\
& Masculino & 2 \\
\cline { 2 - 3 } & 6 & 1 \\
Titulação & 15 & 1 \\
& 17 & 1 \\
\cline { 2 - 3 } Tempo de atuação no ponto da RAPS & Graduação & 1 \\
(em anos) & Mestrado & 2 \\
\cline { 2 - 3 } & 3 & 2 \\
\hline
\end{tabular}

Tabela 1 - Caracterização dos fisioterapeutas participantes da pesquisa Fonte: dados da pesquisa, 2018.

\section{Forma de inserção dos fisioterapeutas na RAPS}

Os três fisioterapeutas participantes da pesquisa entraram na RAPS por meio de concurso público. Todavia, nenhum dos participantes imaginava atuar com saúde mental, uma vez que o concurso realizado era inespecífico, conforme expõem os relatos dos sujeitos.

"Quando eu cheguei aqui eu não sabia o que fazer e nem a direção sabia o que fazer comigo" (F1).

"Nunca imaginei trabalhar com saúde mental" (F2).

"Não tinha ideia do que veria. Não sabia o que esperar" (F3).

Os participantes relataram ausência de experiências pregressas em saúde mental, suas áreas de atuação se concentravam em outras áreas da fisioterapia, como traumato-ortopédica e neurofuncional. Além disso, as pesquisas desvelaram que a inserção da fisioterapia no sistema público ainda é muito limitada, visto que menos da metade dos municípios brasileiros contam com inserção de fisioterapeutas na atenção primária (NÓBREGA, M. et al., 2017; SOUZA; SANTOS 2017; TAVARES; COSTA; DRIUSSO, 2018;). Esses dados são ainda mais preocupantes quando se verifica a inserção no contexto da atenção psicossocial, razão pela qual se justifica a inexperiência ou até mesmo desconhecimento desse campo de atuação no âmbito da fisioterapia. 
O estudo de Ribeiro e Flores-Soares (2015) aponta que os próprios gestores dos sistemas de saúde desconhecem a atuação da fisioterapia, prevalecendo a lógica da visão reabilitadora de desordens osteomusculares. Possivelmente essa compreensão equivocada do papel do fisioterapeuta seja a razão da baixa inserção desses profissionais na atenção psicossocial, o que justifica a necessidade de se evidenciar as contribuições da fisioterapia nas diferentes áreas de atuação, uma vez que até os próprios profissionais desconhecem suas possibilidades de atuação, conforme demonstra a fala dos participantes da pesquisa.

A presença do fisioterapeuta como integrante da equipe multiprofissional de saúde mental tem sido hodiernamente recomendada, pois os recursos fisioterapêuticos impactam positivamente na resposta ao tratamento dos sujeitos em sofrimento psíquico, o que não foi uma realidade nesta pesquisa, visto que há apenas três fisioterapeutas inseridos na RAPS. A evolução do transtorno mental, bem como a ação prolongada dos tratamentos com psicotrópicos, prejudicam tanto as atividades de vida diária dos indivíduos como também as relações interpessoais. Os efeitos positivos da fisioterapia nesses aspectos são destacados pelos próprios sujeitos em sofrimento psíquico que veem a fisioterapia como uma modalidade de tratamento benéfica (HOLANDA et al., 2018; SILVA, S.; PEDRÃO; MIASSO, 2012).

\section{Formação dos fisioterapeutas para o cuidado em saúde mental}

Os relatos dos sujeitos participantes da pesquisa desvelaram que em nenhum momento durante sua graduação obtiveram conhecimentos sobre a atuação da fisioterapia na atenção psicossocial. Por isso, um dos participantes relatou que precisou buscar esse conhecimento que nunca teve por meios próprios para conseguir atuar no cuidado em saúde mental.

"Não se vê abordagem de saúde mental na graduação, não recebi nenhuma capacitação para trabalhar em saúde mental" (F3).

"Quando eu cheguei aqui eu não sabia o que fazer[...]. Precisei frequentar as aulas junto com os médicos residentes em psiquiatria para entender $o$ que era a psiquiatria, entender os efeitos dos medicamentos $e$ as características das doenças" (F1).

Corroborando os dados encontrados, estudos apontam que os profissionais de saúde reconhecem seu despreparo para o cuidado em saúde mental por questões de insegurança e medo frente às situações de crise, uma vez que não são preparados para essa realidade ao longo de sua formação acadêmica (MAGALHÃES; RIBEIRO, 2020; MARTINHAGO; OLIVEIRA, 2012). Outra situação desvelada é que apenas um dos três participantes da pesquisa recebeu capacitação por parte da instituição em que trabalhava para o cuidado em saúde mental. 
O fisioterapeuta adquire competências e habilidades ao longo de sua formação acadêmica que o qualificam para atuação em todos os níveis de atenção do sistema de saúde (MAGALHÃES; RIBEIRO, 2020; RIBEIRO; FLORES-SOARES, 2015). Percebeu-se na análise das grades curriculares dos seis cursos de graduação em Fisioterapia das instituições de ensino superior na região da Grande Florianópolis que o tempo de formação do curso varia de 4 a 5 anos e apenas um dos currículos menciona claramente a oferta de disciplina teórica que aborda o cuidado em saúde mental, corroborando os estudos de E. Silva (2015) e Magalhães e Ribeiro (2020), que apontam a escassa oferta de formação no contexto da saúde mental nos cursos de graduação em Fisioterapia.

Um estudo correlato (SILVA, N. et al., 2013) indica que existem entraves na formação acadêmica dos profissionais de Saúde em função da fragmentação do ensino, que diverge da integralidade do cuidado prevista pela RAPS. Destarte, o modelo biomédico curativista ainda é muito evidente nos centros de formação em Saúde. Esse resultado evidencia fortemente a urgência necessária da reorganização curricular, para que assim os fisioterapeutas saiam da graduação com uma melhor formação e preparo para o cuidado em saúde mental, em concordância com os dados encontrados nesta pesquisa que evidenciou a falta de formação dos fisioterapeutas para atuação na atenção psicossocial.

Nesse sentido, Alves, Dourado e Cortes (2013) apontam que há necessidade de uma visão ampliada para o cuidado em saúde mental para que ele seja efetivamente integral, conforme prevê a RAPS, que abrolhou para ser uma interface na efetivação do modelo substitutivo ao modelo manicomial, possibilitando a reinserção social dos sujeitos. Assim, torna-se fundamental o desenvolvimento de habilidades para a prática em saúde mental, possibilitando a formação de vínculo e afinidade com a área desde a graduação. Mostra-se necessário, portanto, uma reorganização dos currículos da graduação de forma a contemplar a oferta de disciplinas que capacitem o graduando para a atuação na atenção psicossocial, visto que os resultados da pesquisa mostram a oferta em apenas uma instituição de ensino superior.

O desenvolvimento de práticas em campo durante o processo de formação profissional é essencial, pois favorece não só o aprimoramento profissional para a práxis, como também possibilita interação com a equipe multiprofissional envolvida no atendimento dos indivíduos. E pela fala dos participantes da pesquisa fica clara a falta que essa vivência faz para nortear as ações de cuidado. As vivências sobre saúde mental durante a formação acadêmica agregam experiências salientes às carreiras dos profissionais, que são essenciais para o tratamento no modelo de atenção psicossocial que prevê um cuidado crítico e reflexivo pautado na integralidade do indivíduo (FREITAS; PIVETTA, 2017; MAGALHÃES; RIBEIRO, 2020; MUYLAERT; DELFINI; REIS, 2015). 
Assim, destaca-se que o desenvolvimento da prática profissional valoriza-se pelo grau de investimento que é destinado aos profissionais a partir de capacitações, conforme propõem Macedo et al. (2017), o que neste estudo não foi uma realidade, considerando que apenas um dos participantes efetivamente foi capacitado para o cuidado em saúde mental em sua prática laboral. Todavia, salienta-se que é indispensável um conhecimento teórico prévio sobre saúde mental para o norteamento das práticas na atenção psicossocial, o que reforça a necessidade de ampliar a oferta de ensino sobre saúde mental na graduação de Fisioterapia.

\section{CONSIDERAÇÕES FINAIS}

Este estudo mostrou que a inserção da fisioterapia na RAPS no município pesquisado ainda não é uma realidade. Evidenciou-se, assim, que há necessidade de se elucidar as contribuições da fisioterapia no cuidado em saúde mental para ampliar a oferta desse profissional, cujo propósito é prevenir e reabilitar as desordens osteomioarticulares possíveis ou presentes nos sujeitos em sofrimento psíquico. Para que essa inserção seja uma realidade, é necessário ampliar a discussão sobre a atuação do fisioterapeuta na atenção psicossocial nos próprios ambientes de formação desse profissional.

Ressaltou-se também a necessidade de se ter um melhor preparo dos fisioterapeutas, durante a formação acadêmica, para o cuidado em saúde mental, com a oferta de disciplinas específicas para essa área. A pesquisa mostrou que a vivência precoce da abordagem de saúde mental contribui favoravelmente para um melhor enfrentamento futuro das inseguranças frente ao cuidado aos indivíduos em sofrimento psíquico. Desse modo, sugerese que haja uma reorganização dos currículos do curso de Fisioterapia, ofertados pelas instituições de ensino superior da Grande Florianópolis, no sentido de incluir o cuidado em saúde mental no seu rol de disciplinas.

Do mesmo modo, recomenda-se que as instituições de cuidado em saúde mental realizem um melhor preparo do profissional fisioterapeuta, para a atuação no âmbito psicossocial, por meio da educação permanente em Saúde, pois esse preparo é essencial para empoderar os profissionais para o cuidado singular e integral que os sujeitos em sofrimento psíquico necessitam.

Diante das limitações em encontrar estudos que abordem a formação, inserção e atuação da fisioterapia na RAPS, bem como no cuidado em saúde mental, e, ainda, diante da escassez de fisioterapeutas atuando no cuidado em saúde mental no município pesquisado, sugere-se que novos estudos sejam realizados com uma abrangência maior a fim de se verificar se essa é uma realidade nacional ou apenas uma realidade local. 


\section{REFERÊNCIAS}

ALVES, Haiana Maria de Carvalho; DOURADO, Lidiane Bento Ribeiro; CORTES, Verônica da Nova Quadros. A influência dos vínculos organizacionais na consolidação dos centros de atenção psicossociais. Ciência \& Saúde Coletiva, Rio de Janeiro, v. 18, n. 10, p. 2965-2975, 2013. ISSN 1413-8123. DOI: https://doi.org/10.1590/S1413-81232013001000021.

AMORIM, Jamilly Fernandes; FERREIRA, Juliana Barros; SOUZA, Lorenilson Cabral Santos; CARVALHO, Tiago de Souza; MORAIS, Karla Cavalcante Silva de. Percepção dos fisioterapeutas sobre sua atuação no núcleo de apoio à saúde da família. Revista InterScientia, João Pessoa, v. 5, n. 1, p. 105-115, 2017.

BIZARRO, Jean Carlos Müller da Silva. Trajetória dos profissionais da Rede de Atenção Psicossocial: da formação à educação permanente. 2016. Monografia (Graduação em Enfermagem) - Faculdade de Enfermagem, Universidade Federal de Santa Catarina, Florianópolis, 2016. Disponível em: https://grupoapis.paginas.ufsc.br/files/2015/05/TCCJEAN-VERS\%C3\%830-FINAL-PARA-CD-EM-19-07-2016-terezinha-final.pdf. Acesso em: 1 abr. 2020.

BRAGA, Denis Conci; BORTOLINI, Silvia Mônica; PEREIRA, Thiago Gonçalves; HILDEBRANDO, Rafael Bruno; CONTE, Talita Aparecida. Uso de psicotrópicos em um município do meio oeste de Santa Catarina. Journal of the Health Science Institute, v. 34, n. 2, p. 108-113, 2016.

BRASIL. Constituição da República Federativa do Brasil de 1988. Brasília: Congresso Nacional, 1988.

BRASIL. Lei n. 8.080, de 19 de setembro de 1990. Dispõe sobre as condições para a promoção, proteção e recuperação da saúde, a organização e o funcionamento dos serviços correspondentes e dá outras providências. Diário Oficial da União, Brasília, set. 1990a. Disponível em: https://www.planalto.gov.br/ccivil_03/leis/I8080.htm. Acesso em: 15 mar. 2020.

BRASIL. Ministério da Saúde. ABC do SUS: doutrinas e princípios. Brasília, 1990b. Disponível em: https://pesquisa.bvsalud.org/bvsms/resource/pt/mis-2625. Acesso em: 15 mar. 2020.

BRASIL. Ministério da Saúde. Reforma Psiquiátrica e Política de Saúde Mental no Brasil. Documento apresentado à Conferência Regional de Reforma dos Serviços de Saúde Mental: 15 anos depois de Caracas. OPAS. Brasília, 2005. Disponível em: http://bvsms.saude.gov.br/bvs/publicacoes/Relatorio15_anos_Caracas.pdf. Acesso em: 17 mar. 2020.

BRASIL. Ministério da Saúde. Conheça a RAPS - Rede de Atenção Psicossocial. Brasília, 2013. Disponível em:

http://bvsms.saude.gov.br/bvs/folder/conheca_raps_rede_atencao_psicossocial.pdf. Acesso em: 17 mar. 2020.

BÜCHELE, Fátima; LAURINDO, Dione Lucia Prim; BORGES, Vanessa Freitas; COELHO, Elza Berger Salema. A interface da saúde mental na atenção básica. Revista Cogitare 
Enfermagem, Curitiba, v. 11, n. 3, p. 226-233, 2006. ISSN 2176-9133. DOI: http://dx.doi.org/10.5380/ce.v11i3.7308.

CASTRO, S. S.; CIPRIANO JUNIOR, G.; MARTINHO, A. A Fisioterapia no Programa de Saúde da Família: uma revisão e discussões sobre a inclusão. Revista Fisioterapia em Movimento, Curitiba, v. 19, n. 4, p. 55-62, 2006.

COSTA, Ricardo Cesar Rocha. Descentralização, financiamento e regulamentação: a reforma do Sistema Público de Saúde no Brasil durante a década de 1990. Revista de Sociologia e Política, Curitiba, n. 18, p. 49-71, 2002. ISSN 0104-4478. DOI: https://doi.org/10.1590/S010444782002000100005.

DELIBERATO, Paulo César Porto. Fisioterapia Preventiva: fundamentos e aplicações. São Paulo: Manole, 2002.

FREITAS, Caroline Silva de; PIVETTA, Hedioneia Maria Foletto. Fisioterapia na Atenção Básica: um relato de experiência. Revista Científica de Extensão, Santa Maria, v. 3, n. 1, p. 58-75, 2017. ISSN 2447-1151. DOI: https://doi.org/10.5902/2447115123049.

HOLANDA, Rose Lidice; NASCIMENTO, Emanuela Bezerra do; GILO, Hinkilla dos Santos; SILVA JUNIOR, Roque Ribeiro da; ALMEIDA, Maria Tereza Ribeiro de; CERDEIRA, Denilson de Queiroz. Fisioterapia e saúde mental: a percepção dos usuários no cuidado da pessoa em sofrimento psíquico. Revista Expressão Católica Saúde, Quixadá, v. 2, n. 2, p. 45-52, 2018. ISSN 2526-964X. DOI: http://dx.doi.org/10.25191/recs.v2i2.2072.

MACEDO, João Paulo; ABREU, Mariana Marinho de; FONTENELE, Mayara Gomes; DIMENSTEIN, Magda. A regionalização da saúde mental e os novos desafios da Reforma Psiquiátrica brasileira. Revista Saúde e Sociedade, São Paulo, v. 26, n. 1, p. 155-70, 2017. ISSN 1984-0470. DOI: http://dx.doi.org/10.1590/s0104-12902017165827.

MAGALHÃES, Murilo Nunes de; RIBEIRO, Mara Cristina. Percepção de discentes de Fisioterapia sobre sua formação acadêmica em saúde mental. Revista Docência do Ensino Superior, Belo Horizonte, v. 10, p. 1-16, 2020. ISSN 2237-5864. DOI: https://doi.org/10.35699/2237-5864.2020.14800.

MARTINHAGO, Fernanda; OLIVEIRA, Walter Ferreira de. A prática profissional nos Centros de Atenção Psicossocial II (CAPS II), na perspectiva dos profissionais de saúde mental de Santa Catarina. Saúde em Debate, Rio de Janeiro, v. 36, n. 95, p. 583-594, 2012. ISSN 0103-1104. DOI: http://dx.doi.org/10.1590/S0103-11042012000400010.

MUYLAERT, Camila Junqueira; DELFINI, Patricia Santos de Souza; REIS, Alberto Olavo Advincula. Relações de gênero entre familiares cuidadores de crianças e adolescentes de serviços de saúde mental. Physis: Revista de Saúde Coletiva, Rio de Janeiro, v. 25, n. 1, p. 4158, 2015. ISSN 0103-7331. DOI: http://dx.doi.org/10.1590/S0103-73312015000100004.

NÓBREGA, Carolina Bezerra Cavalcanti; HOFFMANN, Rosana Helena Schllitler; PEREIRA, Antonio Carlos; MENEGHIM, Marcelo de Castro. Financiamento do setor saúde: uma retrospectiva recente com uma abordagem para a odontologia. Ciência e Saúde Coletiva, Rio 
de Janeiro, v. 15, p. 1763-1772, 2010. Supl. 1. ISSN 1413-8123. DOI:

http://dx.doi.org/10.1590/S1413-81232010000700088.

NÓBREGA, Maria do Perpétuo Socorro de Souza; DOMINGOS, Alessandra Matheus;

SILVEIRA, Ana Stella de Azevedo; SANTOS, Jussara Carvalho dos. Tecendo a Rede de Atenção Psicossocial Oeste do município de São Paulo. Revista Brasileira de Enfermagem, Brasília, v. 70, n. 5, p. 1016-1023, 2017. ISSN 0034-7167. DOI: http://dx.doi.org/10.1590/0034-71672016-0566.

PAULI, Keli; CAMPOS, Renata. A inserção do fisioterapeuta na equipe multiprofissional do Centro de Atenção Psicossocial. Revista Fisioterapia e Saúde Funcional, Fortaleza, v. 5, n. 1, p. 14-22, 2016.

PITTA, Ana Maria Fernandes. Um balanço da Reforma Psiquiátrica brasileira: instituições, atores e políticas. Ciência e Saúde Coletiva, Rio de Janeiro, v. 16, n. 12, p. 4579-4589, 2011. ISSN 1413-8123. DOI: http://dx.doi.org/10.1590/S1413-81232011001300002.

PREFEITURA MUNICIPAL DE SÃO JOSÉ (PMSJ). São José em Dados. Prefeitura Municipal de São José. Disponível em: https://www.saojose.sc.gov.br/index.php/sao-jose/sao-jose-emdados. Acesso em: 1 jun. 2020.

RIBEIRO, Cristina D.; FLORES-SOARES, Maria C. Desafios para a inserção do fisioterapeuta na atenção básica: o olhar dos gestores. Revista de Salud Pública, Bogotá, v. 17, n. 3, p. 379393, 2015. ISSN 0124-0064. DOI: http://dx.doi.org/10.15446/rsap.v17n3.44076.

RIBEIRO SCHNEIDER, Daniela; BUDDE, Cristiane; CASTILLO FLORES, Karla; PEREIRA, Rafael; TERNES TORRES, Eliane Regina. Políticas de saúde mental em Santa Catarina nos anos 1970: vanguarda na psiquiatria brasileira?. História, Ciência, Saúde, Rio de Janeiro, v. 20, n. 2, p. 553-570, 2013.

SILVA, Elizane Coelho. Abordagem de saúde mental na formação em Fisioterapia: concepções de docentes da área. Revista Contexto \& Saúde, ljuí, v. 15, n. 29, p. 69-79, 2015.

SILVA, Nathalia dos Santos; ESPERIDÃO, Elizabeth; CAVALCANTE, Ana Caroline Gonçalves; SOUZA, Adrielle Cristina Silva; SILVA, Kelly Kan Carvalho da. Desenvolvimento de recursos humanos para atuar nos serviços de saúde mental. Texto e contexto - enfermagem, Florianópolis, v, 22, n. 4, p. 1142-1151, 2013. ISSN 0104-

0707. DOI: https://doi.org/10.1590/S0104-07072013000400033.

SILVA, Soraya Batista; PEDRÃO, Luiz Jorge; MIASSO, Adriana Inocenti. O impacto da Fisioterapia na reabilitação psicossocial de portadores de transtornos mentais. Revista Eletrônica Saúde Mental Álcool e Drogas, Ribeirão Preto, v. 8, n. 1, p. 34-40, 2012. ISSN 1806-6976. DOI: https://doi.org/10.11606/issn.1806-6976.v8i1p34-40.

SOUZA, Madlene de Oliveira; SANTOS, Kionna Oliveira Bernardes. Physical therapists role in Family Health Support Center. Fisioterapia em Movimento, Curitiba, v. 30, n. 2, p. 237-246, 2017. ISSN 1980-5918. DOI: https://doi.org/10.1590/1980-5918.030.002.ao04. 
TAVARES, Larissa Riani Costa; COSTA, José Luiz Riani; DRIUSSO, Patricia. Inserção da fisioterapia na atenção primária à saúde: análise do cadastro nacional de estabelecimentos de saúde em 2010. Fisioterapia e Pesquisa, São Paulo, v. 25, n. 1, p. 9-19, 2018. ISSN 23169117. DOI: https://doi.org/10.1590/1809-2950/15774625012018.

\section{Jean Carlos Müller da Silva Bizarro}

Mestrando em Enfermagem pela Universidade Federal de Santa Catarina (UFSC). Possui especialização em Enfermagem em Dermatologia pela Unyleya (2020), em Docência do Ensino Superior pela Faculdade Futura (2018) e em Enfermagem Estética pela Unyleya (2018). É graduado em Enfermagem pela UFSC (2016) e em Fisioterapia pelo Instituto de Ensino Superior da Grande Florianópolis (2019).

drjeanbizarro@hotmail.com

\section{Eliara Ten Caten Martins}

Doutoranda em Ciências do Movimento Humano pela Universidade do Estado de Santa Catarina (UDESC). Possui mestrado em Ciências do Movimento Humano, também pela UDESC (2015) e graduação em Fisioterapia pela Universidade Regional do Noroeste do Estado do Rio Grande do Sul (UNIJUI). É docente no curso de graduação em Fisioterapia do Instituto de Ensino Superior da Grande Florianópolis (IESGF).

eliara.martins@hotmail.com 